\title{
Computation and evaluation of scheduled waiting time for railway networks
}

\author{
A. Landex \\ Department of Transport, Technical University of Denmark, Denmark
}

\begin{abstract}
Timetables are affected by scheduled waiting time (SWT), which prolongs the travel times for trains and thereby passengers. SWT occurs when a train prevents another train from running at the necessary speed. The SWT affects both the trains and the passengers in the trains. The passengers may be further affected due to longer transfer times to other trains.

SWT can be estimated analytically for a given timetable or by simulation of timetables and/or plans of operation. The simulation of SWT has the benefit of making it is possible to examine the entire network. This makes it possible to improve the future timetable by analyzing different timetables and/or plans of operation. This article presents methods to examine SWT by simulation for both trains and passengers in entire railway networks.

Keywords: scheduled waiting time, timetable, passenger delay, simulation, railway network.
\end{abstract}

\section{Introduction}

When planning timetables for trains, it is often desirable to have more and faster trains along the same line, providing it is a good business case. However, in the timetabling process it is often not possible to fulfil the planning objectives due to capacity constraints. Instead, it is often necessary to reduce the number of trains and/or homogenize the operation by reducing the speed of some trains (planned delays). This creates a conflict between the different planning objectives. For instance, it might not be possible to operate as many fast trains and/or the fast trains as fast as wanted, because fast trains catch up with slower freight/regional trains. 
If the market demand for fast trains is very high compared to that of freight/ regional trains, it might be decided to give the fastest trains a higher priority than the freight/regional trains by running fewer freight/regional trains and/or allowing the freight/regional trains to be overtaken by the faster trains.

The reduced speed due to fast trains catching up with slower trains and additional waiting/dwell time at stations due to overtakings is denoted scheduled waiting time (SWT). The amount of SWT can according to [1] be used as a measure for quality. This is because SWT gives an indication of the extent of conflicts between the planning objectives and the means of action.

SWT depends on the given infrastructure and timetable. It results in longer travel times for trains and passengers. Passengers can be further affected if the needed transfers to/from other trains have long (scheduled) waiting times due to too many interdependencies in the timetable and/or infrastructure.

The article shows how SWT can be considered for trains and passengers respectively. First, SWT for trains (section 2) and for passengers (section 3) is presented. Then it is explained how to calculate SWT for trains (section 4) and passengers (section 5). Section 6 discusses how calculation of SWT can be used to improve the timetables and operation before section 7 draws up the conclusions.

\section{Scheduled waiting time for trains}

When the railway operation results in high capacity consumption, the speed of fast trains must adapt to that of the slower trains, cf. figure 1 (left). This will increase the running time (SWT) for these trains that could run at higher speeds if they were not hindered by other trains. Alternatively, it might be possible to adapt the slower (regional) trains to the faster (intercity) trains by e.g. omitting stops or in the longer term changing the existing trains for trains with better acceleration.

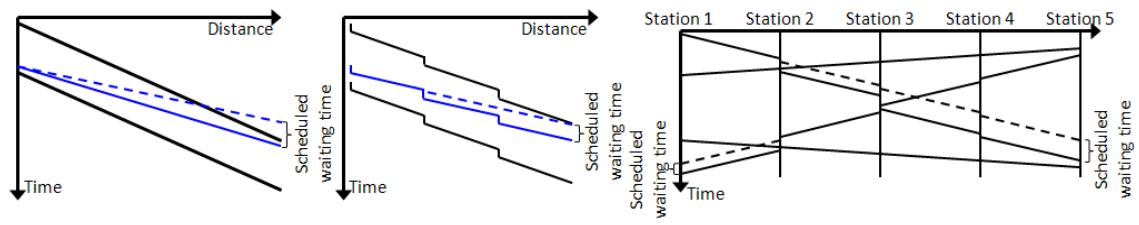

Figure 1: Scheduled waiting time for trains. Based on [2-4].

If the SWT is high (on double track lines), it might be decided to use this time, or part of it, to include extra stops for the fastest train services, cf. figure 1 (middle). In this way the planned timetable has trains with more stops than desired in the wanted timetable. However, it is difficult to evaluate SWT when it, or some of it, has already been converted into additional stops as it is difficult/impossible to identify the stops that have been added to the timetable.

For single track lines, the location of the crossing stations can result in additional dwell time (i.e. SWT), cf. figure 1 (right). 
Pachl [5] states that SWT for trains can be added to the dwell time at stations and to the running time. In Norway, travel time is extended by scheduled waiting time at the railway lines in the suburbs [6]. In The Netherlands, the stations at The Hague (The Hague Holland Spoor and The Hague Central Station) are examples of stations where the dwell time has been extended due to conflicts with other trains [7].

If the traffic demand at the intermediate stops is low, passengers who do not use the stops will experience prolonged travel time due to additional stops. In addition, as a result of the longer travel time, train operating companies might need more trains, and hence more crew, to obtain the same train frequency. Ultimately, the slower travel time can result in a lower frequency of the trains which then reduce the SWT.

\section{Scheduled waiting time for passengers}

SWT for passengers occurs when the travel time is prolonged compared to that in the originally wanted timetable. Therefore, SWT for the trains affects the passengers too. This is because the interdependencies in the railway network prolong the travel time (in the train) and reduce the degrees of freedom in the timetable, which potentially reduces the frequency. However, SWT for passengers also includes transfers. Not all transfers in (larger) public transport networks are well-planned transfers, as improving one transfer due to network effects might worsen others.

Timetable alternatives for a simple railway network for the situations with and without a planned transfer at 'Stop B' can be seen in table 1. Although the running times of the individual trains are unchanged, the travel time for passengers between 'Stop D' and 'Stop C' varies depending on the transfer time at 'Stop B'.

For passengers travelling from 'Stop D' to 'Stop C', timetable scenario 1 in table 1 results in a journey time of 16 minutes, of which 8 minutes is transfer time. However, if timetable scenario 2 in table 1 is used, the journey time would be 26

Table 1: Timetable scenarios between 'Stop D' and 'Stop C' (- indicates trains not serving the station and gray cells indicate the route of the passengers).

\begin{tabular}{r|ccc|ccc|ccc}
\hline Time of departure $\searrow$ & \multicolumn{3}{|c|}{ Scenario 1 } & \multicolumn{3}{|c|}{ Scenario 2 } & \multicolumn{3}{c}{ Scenario 3 } \\
\hline Train $\rightarrow$ & A1 & B1 & B2 & A1 & B1 & B2 & A1 & B1 & B2 \\
\hline Stop D & $\mathbf{2}$ & - & - & $\mathbf{1 2}$ & - & - & $\mathbf{8}$ & - & - \\
Stop A & - & 8 & 28 & - & 8 & 28 & - & 8 & 28 \\
Stop B & $\mathbf{6}$ & $\mathbf{1 4}$ & 34 & $\mathbf{1 6}$ & 14 & $\mathbf{3 4}$ & $\mathbf{1 2}$ & $\mathbf{1 4}$ & 34 \\
Stop C & - & $\mathbf{1 8}$ & 38 & - & 18 & $\mathbf{3 8}$ & - & $\mathbf{1 8}$ & 38 \\
\hline Total time D $\rightarrow$ C & \multicolumn{4}{|c|}{16 minutes } & \multicolumn{3}{|c|}{26 minutes } & \multicolumn{3}{c}{10 minutes } \\
\hline
\end{tabular}


minutes, of which 18 minutes is transfer time, as the corresponding train leaves 'Stop B' only 2 minutes before the train from 'Stop' D arrives at the station.

It is possible to reduce the transfer time in both scenario 1 and 2 and thereby reduce the journey time for passengers travelling from 'Stop D' to 'Stop C'. By reducing the transfer time, the train from 'Stop A' will depart from 'Stop B' 2 minutes after the train from 'Stop D' has arrived. This will ensure sufficient transfer time. This results in a travel time from 'Stop D' to 'Stop C' of 10 minutes (Scenario 3 in table 1). The extra travel time in scenario 1 and 2 (6 minutes and 16 minutes) is SWT for the passengers.

The example in table 1 is straightforward to overview, but according to [8], the reduction of transfer times becomes more complex for more complex networks. Figure 2 shows a journey with two transfers. In the beginning and in the end of the journey there are train services with 20-minute frequency but in between there is a 5-minute frequency train service. By examining the transfers independently, it can be seen that there are short transfers at both stops, but the passengers in the example on the left in figure 2 will not have short transfer time at the second station due to the long waiting time, whereas there is a short transfer time in the example on the right in figure 2 .
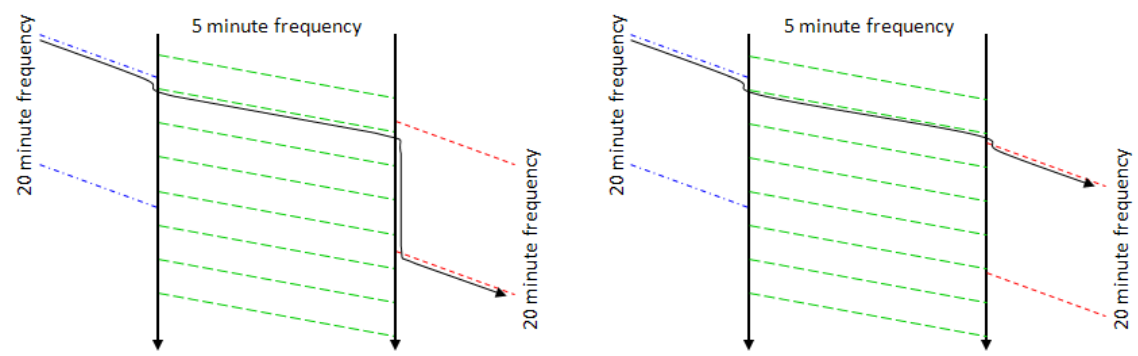

Figure 2: Journey with two transfers: long transfer time (left) and well-planned transfer time (right). Based on [4].

Due to the dependency on the characteristics of the infrastructure and the timetables, the SWT for the passengers can be estimated as the (additional) time the passengers have to spend in the system. This measurement for the SWT for passengers is similar to the SWT measurement for the trains but includes the passengers' waiting time at the station(s).

In the literature, there are various studies where scheduled transfer time, as a part of the SWT for passengers, is attempted to be minimized by changing the time schedules. For instance, [9] minimize the transfer waiting time in a railway system and [10] minimize the transfer waiting time for the bus-train relations for the entire public transport network of Copenhagen. However, these models consider only one transfer, and not the passenger's total journey, why the total SWT for passengers is underestimated. 


\section{Calculation of scheduled waiting time for trains}

SWT can according to $[11,12]$ be estimated analytically for a given timetable but it can also be estimated by simulating plans of operation. The Danish developed SCAN model (Strategic Capacity Analysis of Network) is a strategic tool to calculate capacity in a railway network $[13,14]$. The tool simulates random regular interval timetables (see $[15,16]$ for classification of timetables) and calculates their SWT. For this, SCAN uses the infrastructure (on the meso level - see [17] for the aggregation levels of infrastructure data), the plan of operation (i.e., the number of trains within each category and their stop pattern) and the main dynamics of the rolling stock. The workflow of calculating SWT using SCAN is [14]:

1. Prepare the model (build up infrastructure, key in dynamics of rolling stock, and enter a plan of operation).

2. Calculate minimum running time and kilometers of operation.

3. Generate regular interval timetables by random departure times for the first departure for each train system (at the first station). The following departure times for each train system are determined by the frequency. In this way a number of different timetables are generated. In this stage there may be conflicts between trains.

4. Synchronic simulation of each timetable by a discrete simulation model where the priority of the trains determines which trains run first. The result of the simulation is a conflict-free timetable for how trains can be operated.

5. Calculate running time and SWT (difference between simulated running time and minimum running time) for each timetable.

The flow of calculating the SWT can be seen in figure 3.

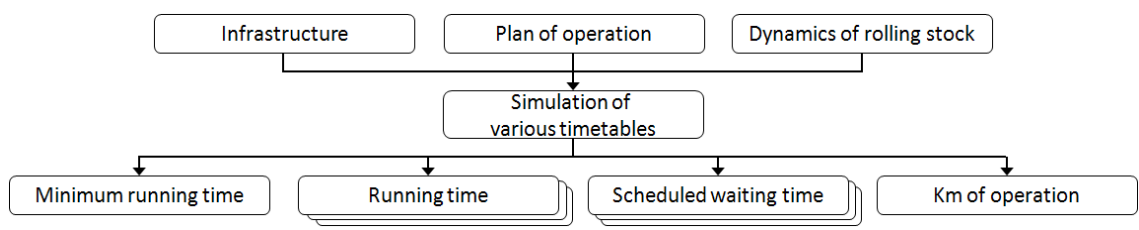

Figure 3: Calculation of SWT in the SCAN model [4].

Examining a large number of different timetables based on the same plan of operation will result in different SWTs. These different SWTs can then be sorted according to the SWT as shown in figure 4. It is then possible to see the span in SWT and choose the timetable that has the lowest scheduled waiting time and still fulfils other potential requirements of the timetable, e.g., possible transfers between trains. Consequently, the final (chosen) timetable is not necessarily the timetable with the lowest SWT. Therefore, [14] suggests using the $25 \%$ percentile to describe a satisfactory quality of operation, and thereby the expected SWT of the plan of operation. 
A problem with the SCAN model is that timetable supplements are not included. Therefore, the model can be used only to evaluate the plan of operation at the strategic level. Alternatively, the North American Train Performance Calculator (TPC) can be used to generate a large number of timetables, which can be investigated. The workflow in the TPC model is (based on [18]):

1. Prepare the model (build up infrastructure, key in dynamics of rolling stock and train services).

2. Generate timetables randomly by choosing random departure times for all trains.

3. Simulation of each timetable to generate conflict-free timetables.

Based on the results of the TPC-model, it is possible to calculate the actual running times of the timetables. To examine SWT it is necessary to calculate the minimum running time of the train services too. The timetables can then be ranked by SWT as in figure 4 .

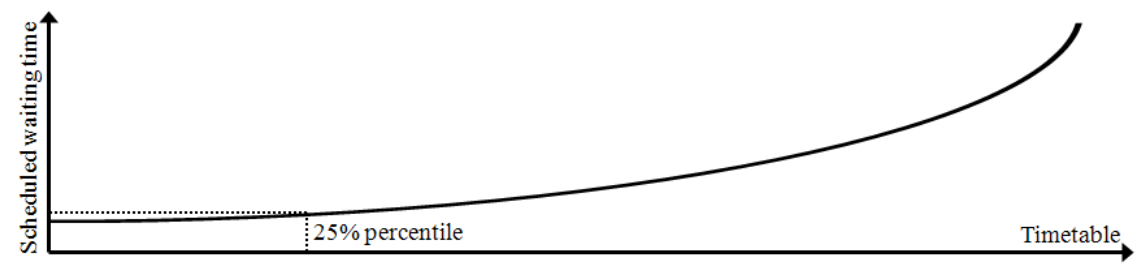

Figure 4: Sorting the timetables according to the SWT. Based on [3].

The major differences between the TPC and SCAN models are that the SCAN model examines randomly generated regular interval timetables, while TPC examines timetables where all the trains are operated randomly; furthermore, SCAN calculates the SWT itself, whereas the calculation has to be done manually in the TPC model.

In North America, the trains are (as described in [5, 19]) operated according to a more or less improvised timetable, which is why the TPC model is well suited there. This random operation is possible because only few corridors are dominated by passenger trains. However, in Denmark (and Europe) the operation is mostly based on regular interval timetables; accordingly, the TPC model is less suited to simulate the operation here. The SCAN model simulates regular interval timetables but does not include timetable supplements. To have a better simulation model well suited for analyses in the Danish/European context, the SCAN model should be developed to include timetable supplements, and/or the TPC model should be adapted to examine regular interval timetables.

Generally, simulation models based on future plans of operation are well suited for strategic network analyses, but simulation models has difficulties examining where the capacity problems and SWT, are most severe. Consequently, it is also difficult to examine where the infrastructure should be improved and what effects the improvement will have. Combining microscopic and macroscopic models can, 
however, help in this kind of analysis. Railnet Austria has according to [20] combined microscopic and macroscopic models in the infrastructure planning. Here, future timetables have been developed and evaluated (in this case by the UIC 406 method - [21] describes the UIC 406 methodology) by converting data between the macroscopic and microscopic level; the methodology could be the basis when evaluating SWT for different timetable alternatives.

\section{Calculation of scheduled waiting time for passengers}

SWT for passengers is the delay of the passengers compared with that of the 'optimal' timetable. This definition is very similar to the SWT for trains. However, cases with a small amount of SWT for trains do not necessarily result in a small amount of SWT for passengers, and vice versa. This is because just little SWT for a train might result in a lost transfer for the passengers, but only if the transfer time is tight, otherwise the total travel time remains unchanged.

The SCAN model used to calculate SWT for trains can also be used to calculate SWT for passengers. This is because the output timetables from SCAN can be used as a basis to calculate passenger delays as the difference between the times used in the actual analyzed timetable and the best-analyzed timetable.

Using simulation models, such as SCAN, the risk of delays in the operation, and thus the risk of missing a transfer, is omitted, which makes it difficult to analyze SWT in real and contingency operation. Hence a high risk of missing a connecting train will increase the travel time. Therefore, a timetable without planned transfer(s) might be better than the timetable with planned transfer(s) if the risk of delays is high, as the travel time for the passenger will most likely remain the same.

To reflect the actual operation and take the punctuality of the railway system and thereby the risk of missing a connecting train - into account when calculating SWT for the passengers, it is necessary to simulate the (candidate) timetables. This can be done by 'traditional' simulation where the infrastructure and timetables are built up before simulating the operation with initial delays, cf. figure 5 .

Passenger delay models can based on the output of 'traditional' simulation estimate how much time the passengers spend in the railway system. Therefore, passenger delay models can be used to deduce SWT for passengers. [4] defines different types of passenger delay models of which only the newest generations can be used to estimate SWT.

The different combinations of infrastructures and timetable variants will result in a different amount of SWT in the system. This type of traditional simulation project is time consuming, but by combining microscopic and macroscopic models, the workload of generating and simulating timetables can according to [23,24] be reduced. 


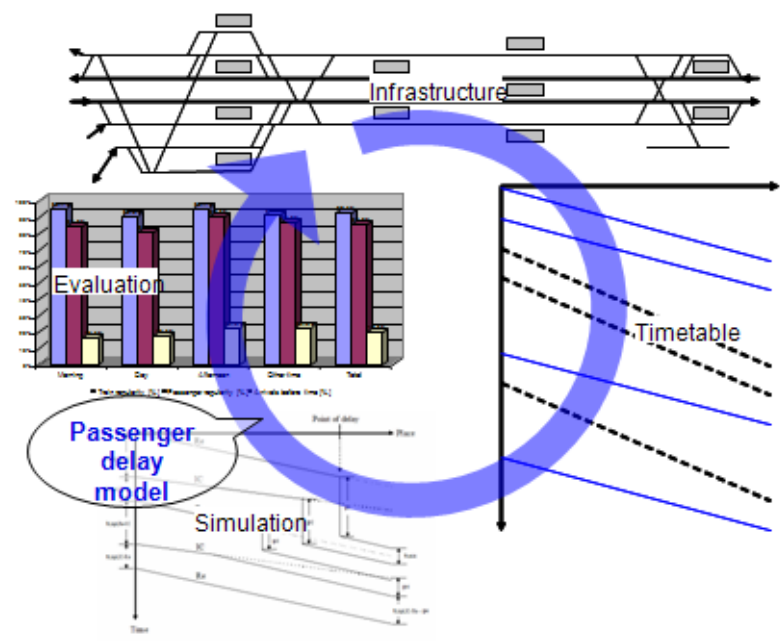

Figure 5: Simulation of railway traffic with passenger delays. Based on [22].

\section{Discussion}

Timetables for railway networks can be improved by examining SWT in the planning process. By examining SWT for different future (candidate) timetables, it is possible to examine different timetable strategies, for example additional overtaking. In the examination, it is possible to evaluate both the time gain for the passengers in the fast train and the time loss for the passengers in the train that is overtaken. The examination of SWT can be done either locally for a single railway line or for the entire system including transfers to/from other trains.

Improving the timetables without taking the risk of train delays during the operation into account can result in an over-optimized timetable for passengers. This is because even small train delays will result in lost transfers for the passengers - or even degenerated schedules. To take common train delays into account, it is recommended to simulate of the timetables with a typical delay distribution. The additional SWT for the passengers can then be calculated based on the simulated timetables. This makes it possible to plan timetables with less SWT for normal operation.

Optimizing SWT is not possible in all types of timetables, for example, in an integrated fixed interval timetable (see $[15,16]$ for classification of timetables) where all trains meet at the same time at stations/hubs throughout the network. In integrated fixed interval timetables the structure of the timetable is fixed when the stations/hubs have been selected. Additionally, SWT has virtually been determined by the chosen stations/hubs as all the trains have to meet at the station/hub and a train is not permitted to leave before the last train has arrived (and the passengers have had time to make a transfer). Therefore, SWT cannot be optimized in integrated fixed interval timetables, but the amount of SWT can be used to 
describe how well the infrastructure can handle the chosen integrated fixed interval timetable. This can be used to examine which improvements in the infrastructure that reduce SWT the most.

In the longer term the simulation approach can also be used by the dispatch centers to decide if a train should wait for a delayed train to obtain the planned transfer. Thus, the simulation of the traffic combined with calculating SWT for trains and passengers can be used to evaluate the consequences of different scenarios. In this way it is possible to improve the operation.

The short-term operation can be improved too by including evaluation of SWT in the planning process. Timetables can be simulated and SWT for both trains and passengers can be calculated so that the best possible timetable is chosen. This approach can also be used when planning timetables for contingency operation, so the best timetable can be used in cases of disrupted operation.

\section{Conclusion}

Railway operation is often affected by scheduled waiting time (SWT) because fast trains (due to infrastructure restrictions) cannot overtake slower trains. This means that additional time - SWT - has to be implemented in the timetable. The article shows how SWT affects both the trains and the passengers in the trains. The article also demonstrates that passengers are further affected by SWT in the case of transfers.

The article illustrates that SWT for trains can be calculated by simulation models such as the Danish SCAN model and the North American TPC model. Based on SWT for trains and passenger delay models the article presents a method to calculate SWT for passengers. The article also demonstrates how it is possible to estimate SWT in the case of delays.

Calculating SWT for candidate timetables makes it possible to test different timetable strategies and choose the best strategy for the final timetable. This can improve the timetables for both the operator(s) and the passengers.

\section{References}

[1] Handstanger, A.C.T., Scheduled waiting time from crossing on single track railway lines. Ph.D. thesis, Norwegian University of Science and Technology, 2009.

[2] Salling, K.B. \& Landex, A., Computer based ex-ante evaluation of the planned railway line between Copenhagen and Ringsted by use of a new Decision Support System named COSIMADSS. CompRail X, p. 65, 2006.

[3] Landex, A. \& Nielsen, O.A., Network effects in railway systems. European Transport Conference, 2007.

[4] Landex, A., Methods to estimate railway capacity and passenger delays. Ph.D. thesis, Technical University of Denmark, 2008. 
[5] Pachl, J., Railway Timetable \& Traffic, Eurail Press, chapter Timetable Design Principles, pp. 9-42, 2008.

[6] Skartsæterhagen, S., Capacity of railway lines (Kapacitet på jernbanestrekninger). Technical report, Institute for Energy Technology, Norway, 1993.

[7] Nie, L. \& Hansen, I.A., System analysis of train operations and track occupancy at railway stations. EJTIR, 5(1), pp. 31-54, 2005.

[8] Klemenz, M. \& Radtke, A., Method and software tool for an optimized passenger orientated connection management. Computers in railways XI, WITpress, p. 3, 2008.

[9] Wong, R.C.W. \& Leung, J.M.Y., Timetable synchronization for mass transit railway. International Conference on Computer-Aided Scheduling of Public Transport (CASPT), 2004.

[10] Pedersen, M.B., Nielsen, O.A. \& Jansen, L.N., Minimizing passenger transfer times in public transport timetables. Conference of Hong Kong Society for Transport Studies: Transportation in the information age, Hong Kong Society for Transport Studies, p. 229, 2002.

[11] Wendler, E., The scheduled waiting time on railway lines. Transportation Research Part B, 41(2), pp. 148-158, 2007.

[12] Wendler, E., Railway Timetable \& Traffic, Eurail Press, chapter Queuing, pp. 106-117. 1st edition, 2008.

[13] Kaas, A.H., Development and practical use of a capacity model for railway networks. Conference on Structural Integrity and Passenger Safety, WITpress, p. 73, 1998.

[14] Kaas, A.H., Methods to calculate capacity of railways (Metoder til beregning af jernbanekapacitet). Ph.D. thesis, Technical University of Denmark, 1998.

[15] Liebchen, C., Periodic Timetable Optimization in Public Transport. Ph.D. thesis, Technical University of Berlin, 2006.

[16] Schittenhelm, B., Identification of timetable attractiveness parameters by an international literature review. Annual Transport Conference at Aalborg University (Trafikdage), 2008.

[17] Gille, A., Klemenz, M. \& Siefer, T., Applying multiscaling analysis to detect capacity resources in railway networks. Computers in railways XI, WITpress, p. 595, 2008.

[18] White, T.A., The development and use of dynamic traffic management simulation in north america. International Seminar on Railway Operations Research, IAROR, 2007.

[19] White, T.A., North american experience with timetable-free railway operation. International Seminar on Railway Operations Modelling and Analysis, IAROR, 2005.

[20] Sewcyk, B., Radtke, A. \& Wilfinger, G., Combining microscopic and macroscopic infrastructure planning models. International Seminar on Railway Operations Modelling and Analysis, IAROR, 2007.

[21] International Union of Railways (UIC), Capacity (UIC code 406), 2004. 
[22] Landex, A. \& Nielsen, O.A., Modelling expected train passenger delays on large scale railway networks. World Congress on Railway Research, 2006.

[23] Kettner, M. \& Sewcyk, B., A model for transportation planning and railway network evaluation. World Congress on Intelligent Transport Systems, 2002.

[24] Kettner, M., Sewcyk, B. \& Eickmann, C., Integrating microscopic and macroscopic models for railway network evaluation. European Transport Conference, 2003. 